Research Article

\title{
Evaluation of Potential Correlation of Piano Teaching Using Edge-Enabled Data and Machine Learning
}

\author{
Sibing Sun \\ School of Music, Harbin Normal University, Harbin 150080, China \\ Correspondence should be addressed to Sibing Sun; ssb@mail.sdufe.edu.cn
}

Received 23 December 2020; Revised 15 January 2021; Accepted 1 February 2021; Published 14 April 2021

Academic Editor: Mian Ahmad Jan

Copyright $\odot 2021$ Sibing Sun. This is an open access article distributed under the Creative Commons Attribution License, which permits unrestricted use, distribution, and reproduction in any medium, provided the original work is properly cited.

\begin{abstract}
Data science has expanded at an exponential growth with the advancement of big data technology. The data analysis techniques need to delve deeper to find valuable information (Sarac 2017). The notion of edge computing is broadly acknowledged. Edgeenabled solutions provide computing, analysis, storage, and control nearer to the edge of the network, which support the efficient processing and decision-making. Machine learning has also attained significant attention in this context due to its flexibility and its ability to provide a variety of supervised, unsupervised, and semisupervised techniques. This research presents a specific model to evaluate the potential correlation of piano teaching using machine learning. The data analysis is performed at the edges of network for efficient results (Tan et al. 2017). The association rule mining technique of machine learning is utilized with the integration of improved $T$-test method. The improved $T$-test is performed for the measurement of association rules and proposed a new measure and influence degree of association rules. It is evident from the results that the use of the degree of influence as a measure of association rules to find the potential relevance of multimedia-assistant piano teaching evaluation data is extremely feasible. It overcomes shortcomings of existing measurement standards and reduces the generation of redundant rules. The existing literature highlights the concepts of evaluation of potential correlation and evaluates the advantages. However, there is a lack of an effective strategy for piano teaching. The proposed model performs efficient calculation and storage. The feasibility and effectiveness of the proposed framework are verified using the analysis of the actual dataset. The verification results show that it is feasible and valuable to find the potential relevance of multimedia-assisted piano teaching evaluation.
\end{abstract}

\section{Introduction}

The emergence of Internet of Things (IoT) and the proliferation of machine learning have improved the way we act and think. Entering the new era of machine learning, an effective strategy for data analysis has become a challenging task. The existing models face challenges to have efficient data analysis and evaluation. Educational technologies (e.g., multimedia technology) improve the popularity of the modern educational system. Multimedia technology improves the quality of teaching in various applications. Among these applications, piano teaching is one such application that has benefitted from using multimedia technology. The use of modern educational technologies in piano teaching has been popularized in recent years. In fact, these technologies have promoted the application of modern education to a new horizon. Due to the continuous development of multimedia technology, various educational theories and teaching models have been put forward, so that multimedia piano teaching also faces some new problems. The intersection of these aspects is the need to scientifically evaluate and analyze the quality and effectiveness of multimedia piano teaching. At present, the existing evaluation system can realize the functions of data entry, query, and statistics but cannot discover the relationships and rules existing in the data and cannot predict the future development trend based on the existing data. Therefore, the big data mining technology based on machine learning is applied to the analysis of multimedia piano teaching evaluation data, which provides decision support for teaching managers, which is of great significance to the improvement of multimedia piano teaching quality [1]. 
The idea of edge computing is gaining prominence with the rise of complex data analysis. Edge-enabled solutions provide efficient processing and control nearer to the edge for scalability and latency management. Edge computing offloading technology uses computing-intensive that is hard to handle by mobile devices. The relocation of jobs to the servers nearby the edge device not only advances the processing capability of the devices. This paper proposes a multimedia-assisted piano teaching model based on big data machine learning. The data analysis is performed at the edges of network for efficient results. The association rule mining technique of machine learning is utilized with the integration of improved $T$-test method. The improved $T$-test is performed for the measurement of association rules and proposed a new measure and influence degree of association rules. The paper proposes an improved $T$-test-based model using the traditional support degree, confidence framework, validity, and relevance. The influence degree model analyzes the influence degree measurement and verifies its effectiveness using a specific case study [2].

\section{Data Analysis and Evaluation of Multimedia- Aided Piano Teaching}

The evaluation of multimedia-assisted piano teaching is not the purpose of evaluation activities. The ultimate goal of evaluation is to improve the work, scientific management, and research decision-making by evaluating the information obtained. This evaluation can be improved by using the edge-commuting. The edge-enabled analysis will improve the efficiency of the data analysis process. The evaluation information data do not spontaneously play a role. It is necessary to properly process the evaluation information along with its feedback. The feedback evaluation information exerts its comprehensive role as much as possible to make the evaluation activity effective. The data analysis factors are described in the coming sections.

\subsection{The Factors Affecting the Quality of Multimedia-Assisted} Piano Teaching. The development of multimedia-assisted piano teaching is a complex systematic project, which is closely related to the corresponding theoretical research, technological progress, teaching practice, and so forth. Its teaching effect is influenced by many factors such as the information literacy of teachers and students, the quality of teaching courseware, and the teaching support platform. At present, multimedia-assisted piano teaching is still in the exploration stage in terms of teaching mode, teaching methods, and teaching strategies. Therefore, through the big data and machine learning technology to analyze the factors affecting the effect of multimedia teaching, it provides various links for improving multimedia-assisted piano teaching. Valuable information is of great significance to improve the quality of multimedia teaching.

2.2. Feedback on Evaluation, Analysis, and Timely Adjustment in Teaching Methods and Processes. In this information society, the efficiency of information transmission and the timeliness of feedback accelerate the process of social development. In the evaluation process, we must allow the advantage of using computer network technology in data processing and information transmission. The computer processing is made more efficient with the help of edge computation where the processing unit is provided with the network edges. The collected data are sorted and analyzed to obtain the distribution state of the data. The characteristics, rules, and relationship of the data is utilized at the edges and useful information is extracted for improving the quality of multimedia-assisted piano teaching and contributing to teaching management. According to the evaluation feedback information, solve various problems in the teaching in time, further optimize various aspects of multimedia-assisted piano teaching, give full play to the advantages of multicoal-assisted piano teaching, and lay the foundation for improving the quality of piano teaching.

\subsection{Teachers' Information Literacy and Teachers' Self-Im-} provement Promotion. Multimedia-assisted piano teaching combines the advantages of multimedia teaching and traditional teaching and plays a big role in improving teaching quality. However, at the same time, it also puts forward higher requirements for the quality and ability of teachers engaged in multimedia piano teaching. In the evaluation process, we must strive to provide teachers with useful information through the orientation, diagnosis, and timely feedback of the evaluation system with the help of edge computing. Through the analysis of the evaluation data, we find out the problems and deficiencies of teachers in all aspects of multimedia piano teaching and help teachers improve their multimedia information, emergency technology, and classroom integration ability to promote the self-improvement and improvement of piano teachers. The evaluation index of multimedia-assisted piano teaching model is shown in Figure 1.

2.4. Guarantee the Reliability of the Evaluation Data. Evaluation is a subjective activity, and the evaluator's attitude is direct or not, which has a direct impact on the evaluation results. Scientific processing of the obtained evaluation data is important and an important issue to ensure the quality of the evaluation. Since the evaluation indicators are derived from the same target, there must be an intrinsic link between various indicators, which also leads to some correlation between the evaluation data of different evaluation indicators. We can find out that through the association rules. The correlation between the evaluation data is used, and it is checked whether the obtained evaluation data are reliable based on the correlation.

\section{Machine Learning-Based Association Rule Metrics}

In this section, the machine learning-based association rule metrics are designed. The overall process is applied to the multimedia-aided piano teaching techniques considered as 


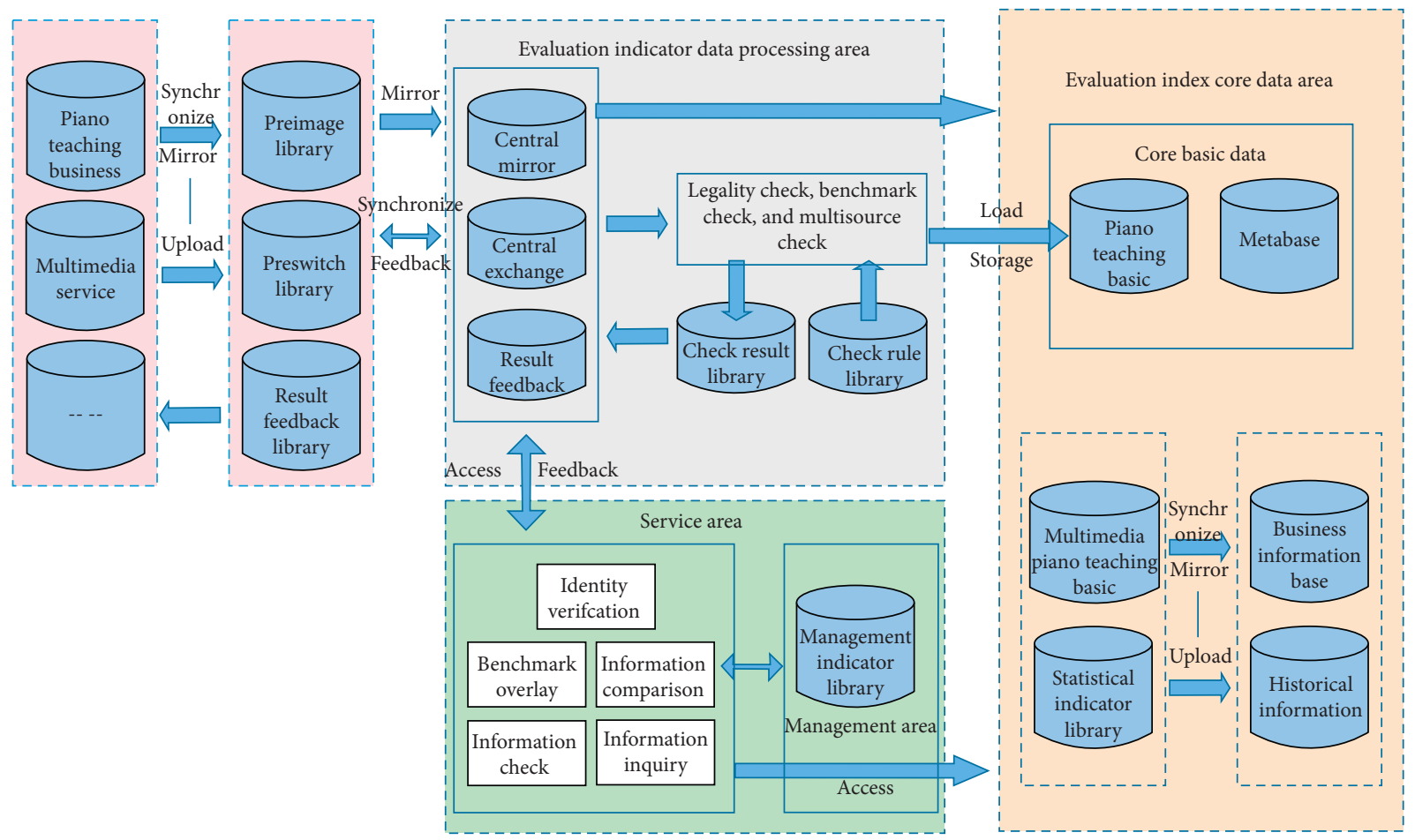

Figure 1: Evaluation index of multimedia-assisted piano teaching model.

the edge of the networks. Initially, the standard is discussed in the context of association rules. A detailed description is provided in the subsection.

3.1. Machine Learning Association Rule Standard. In the past few years, there have been many controversies about the problems of traditional support and credibility models, and many improvements have been proposed. Various new rule evaluation criteria have been proposed and added to the mining algorithm. The generation of association rules is restricted and constrained to obtain more novel and effective association rules. There are broadly two different categories. The first category is to try to find alternatives to the measure of credibility and to improve the objective evaluation method of extending the support threshold limit. The second category is to try to increase the subjective measure. Multimedia-assisted piano teaching data mining process diagram is shown in Figure 2.

Data mining of multimedia-assisted piano teaching data is first performed. The data mining process can be divided into the following steps:

(1) Data cleansing: eliminating noise and data not related to the mining theme.

(2) Data integration: integrating data from multiple data sources.

(3) Data selection: selecting data related to mining topics.

(4) Data transformation: using data such as normalization to transform data into a form suitable for data mining.
(5) Data mining: the core steps to mine knowledge using methods such as classification, fusion, and association rules.

(6) Mode evaluation: evaluation of the effect of the model, the commonly used indicators have accuracy, recall rate, and so on.

(7) Knowledge representation: the model represented by a technically understandable model and the knowledge obtained by the mining presented to the user.

3.2. Relativity. Relativity [3-5] is the ratio of confidence to expected confidence, and its expression is defined as

$$
\text { relativity }(X \Rightarrow Y)=\frac{P(Y \mid X)}{P(Y)}=\frac{P(X Y)}{P(X) P(Y)},
$$

where $P(Y)$ is the expected confidence of the rule $X \Rightarrow Y$, indicating that the transaction in $D$ contains the percentage of $Y$. The relevance of the rule $X \Rightarrow Y$ describes the influence of the item set $X$ on the item set $Y$. When the degree of correlation is equal to 1 , it indicates that $X$ has no effect on the $Y$; that is, $X$ and $Y$ are independent, and the rule is called an irrelevant rule. At 1 o'clock, it indicates that the occurrence of $X$ is lower than the possibility of occurrence of $Y$, and the rule is said to be a negative correlation rule; when it is greater than 1 , it indicates that the appearance of $X$ increases the possibility of occurrence of $Y$, which is called a positive correlation rule [6]. Through the analysis of the correlation, the misleading strong association rules can be effectively filtered out. The relevance definition of association rules $X \Rightarrow Y$ fully considers the directionality of the rules. 


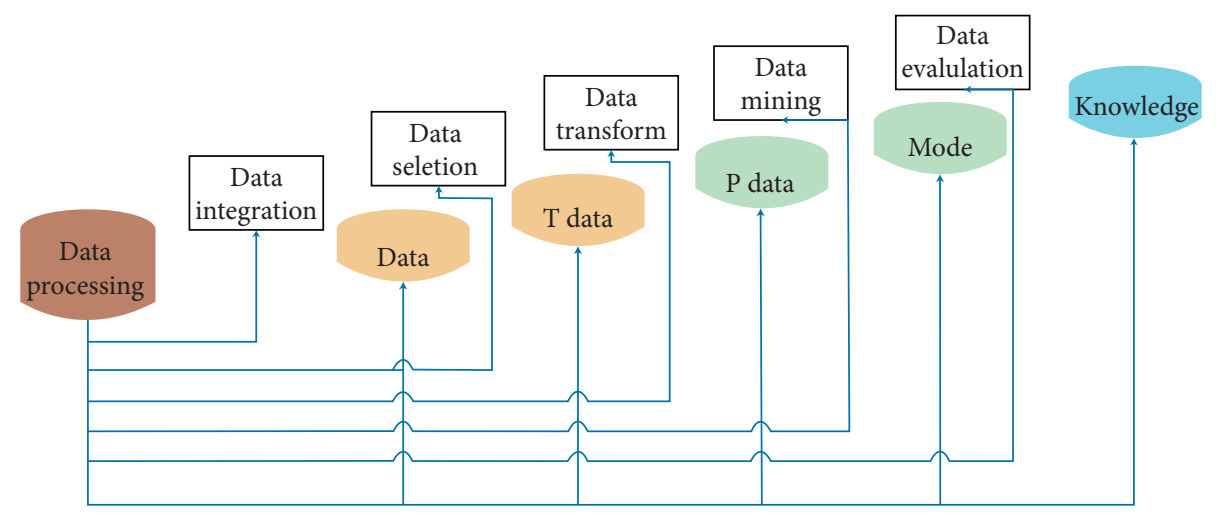

FIGURE 2: Data mining process workflow.

The greater the correlation, the greater the positive correlation between $Y$ and $X$ and the greater the positive effect of $X$ on $Y$; the lesser the correlation (less than 0 ), the greater the intensity of negative correlation between $Y$ and $X$; and the greater the inhibition of $X$ to $Y$, the bigger the effect. This correlation is utilized in the proposed model for data analysis of piano teaching evaluation. In addition, the relativity is computed at the edges of the computing [7].

3.3. Interest Level. The interest of the basis difference is defined as follows:

$$
\text { Interest }(X \Rightarrow Y)=P(Y \mid X)-P(Y) \text {. }
$$

According to the definition of interest, there are three possible scenarios for the metric of interest $(X \Rightarrow Y)$ :

(1) If the degree of interest is $>0$, then $X$ and $Y$ are positively correlated.

(2) If the degree of interest is $<0$, then $X$ and $Y$ are negatively correlated.

(3) If the degree of interest $=0$, then $X$ and $Y$ are irrelevant and independent of each other.

In fact, there are many definitions of the interestingness of the rules. The interestingness is defined in the literature [8-11]:

$$
\text { Interest }(X \Rightarrow Y)=\frac{P(Y \mid X) / P(X)-P(Y)}{\max (P(Y \mid X) / P(X), P(Y))} \text {. }
$$

This definition makes the interest level between -1 and 1 , and the interestingness of a rule is greater than 0 , indicating that the more interested in the rule (the greater its practical value), the more unreasonable the rule, and the more interested people are in the negative rule of the rule (that is, the greater the actual use value of the reverse rule). Among the factors influencing the quality of rules, in addition to the three factors that affect the degree of interest, such as coverage, implementation, and confidence, there are also rule complexity factors. In addition, Alex also analyzed several other factors that may affect the quality and interestingness of the rules, including the size of the rule front, the imbalance of class distribution, the influence of rule attributes, the cost of misclassification, and the classification rules.

3.4. Relative Confidence. The literature [8] introduces the concept of relative confidence. If the probability $P(X)$ is used to represent the percentage of transactions in $D$ containing $X, P(\bar{X})$ is used to indicate that the transaction in $D$ does not contain the percentage of $X, P(Y \mid X)$, which means that $Y$ appears in transaction $D$ when $D$ appears. In the calculation of conditional probability, $P(Y \mid \bar{X})$, when transaction $X$ does not appear, the conditional probability of appearing in $D$ is $Y$. If $X$ is a frequent item set, then min sup $\leq P(X) \leq 1$. The definition of $X \Rightarrow Y$ relative confidence is as follows: if $\min \sup \leq P(X) \leq 1$, relative confidence is $P(Y \mid X)-P(Y \mid \bar{X})$.

If $P(X)=1$ or $P(\bar{X})=0$ and $P(Y)=1$, relative confidence is 1 .

If $P(X)=1$ or $P(\bar{X})=0$ and $P(Y)<1$, relative confidence is 0 .

If $P(X)=1$, this means that each transaction in $D$ contains $X$. If each transaction contains $\mathrm{Y}$ at the same time, the relative confidence is defined as 1; otherwise, the relative confidence is defined as 0 . Confidence due to strong association rules $P(Y \mid X) \geq$ min confidence. Therefore, the value range of relative confidence is $[-1+\min$ confidence, 1$]$.

Introducing a threshold of relative confidence $R_{1}$ and $R_{2}$, they are all positive numbers greater than 0 and less than one. The relative confidence of strong association rules must be one of the following three situations:

$$
\begin{aligned}
& R_{1} \leq \text { relative confidence } \leq 1 \\
& -R_{2}<\text { relative confidence }<R_{1} \\
& -1<\text { relative confidence } \leq-R_{2} .
\end{aligned}
$$

According to the value of relative confidence, strong association rules can be divided into three categories: positive association rules, invalid association rules, and negative association rules [12]. 
3.5. Effectiveness. The concept of validity is introduced in [13]. If $P(X)$ is used to indicate the probability of occurrence of transaction $X, P(\bar{X})$ is the probability that transaction $X$ does not occur. The support and confidence of association rules $X \Rightarrow Y$ can be expressed as $P(X Y)$ and $P(X Y) / P(X)$, respectively. The validity of defining $X \Rightarrow Y$ is

$$
\text { Validity }=\frac{P(X Y)}{P(X)^{*} P(X)}-\frac{P(\bar{X} Y)}{P(\bar{X})^{*} P(\bar{X})} .
$$

The intuitive meaning of the above formula is as follows: validity $=$ (probability of simultaneous occurrence of $X$ and $Y$ in the $D$ database) - (probability of simultaneous occurrence of $Y$ in the $D$ database). Since the value intervals of $P(X Y)$ and $P(\bar{X} Y)$ are both at $(0,1)$, it is obvious that the value range of the validity is $(-1,1)[14]$.

It can be seen from the definition of validity that if the validity of $X$ and $Y$ is less than zero, it means that the probability of occurrence in the $D$ database is less than the probability of occurrence; if the validity of $X \Rightarrow Y$ is equal to zero, the probability of occurrence in the $D$ database is equal to the probability of occurrence [15]. Therefore, when mining association rules, if the validity of $X \Rightarrow Y$ is less than or equal to zero, even if the support and confidence are satisfactory, the extracted association rules are still useless association rules [15].

3.6. Suitability. Considering the possibility of $Y$ appearing when $X$ does not appear and whether $X$ and $Y$ are related, the concept of matching is proposed, which is defined as follows.

For example, $P(X)$ denotes the probability of occurrence of $X, P(Y)$ denotes the probability of $Y, P(X Y)$ denotes the probability of simultaneous occurrence of $X$ and $Y, P(\bar{X} Y)$ denotes the probability of simultaneous occurrence of $\bar{X} Y$, and $P(\bar{X})$ denotes the probability that $X$ does not appear; then, the matching degree of $X \Rightarrow Y$ is defined as follows: (the confidence of $X \Rightarrow Y$ ) - (confidence of $X \Rightarrow Y$ ); namely,

$$
\text { match }=\frac{P(Y \mid X)}{P(X)}-\frac{P(Y \mid \bar{X})}{P(\bar{X})} .
$$

If match $>0$, then $P(X Y)>P(X) P(Y)$, explaining that $X$ and $Y$ have positive correlations.

If match $=0$, then $P(X Y)=P(X) P(Y)$, explaining that $X$ and $Y$ are irrelevant, that is, redundant rules.

If match $<0$, then $P(X Y)<P(X) P(Y)$, declaring that the negative rules of the rules may have research value.

If match $=0$, then $P(X Y)=P(X)=P(Y)$, noting that $X$ and $Y$ appear simultaneously or not in the record set.

As can be seen from the formula, the definition of matching not only includes the relevance factor but also includes the factor of $P(Y)$. Therefore, the degree of matching can fully reflect the validity of the rule $X \Rightarrow Y$.

3.7. Metric Improvement. The matrices can be improved in two different ways that are (1) insufficient measures of some association rules and T-test. A detailed description is provided as follows [16].

3.7.1. Insufficient Measures of Some Association Rules. In the pattern of generating association rules, the classic support and confidence framework are generally used to generate association rules. However, when the framework is used to generate rules, a large number of redundant and irrelevant rules are generated, which affects the user's choice of rules and even misleads the judgment [17]. Many researchers now use a variety of metrics to filter the association rules they have acquired to obtain effective rules. As illustrated in the example shown in the following table, this is a project set of length 2. The well has minimum support and confidence of 0.2 and 0.5 , respectively. A set of transaction data are shown in Table $1[18,19]$.

From the table, it can be said that $I$ and $J$ always appear or do not appear at the same time, which is a valid association rule. When $I \Rightarrow J$, the support and confidence are 0.4 and 1 , respectively. In the network sample, when $G \Rightarrow E$, support and confidence are also 0.4 and $1 . I \Rightarrow J$ and $G \Rightarrow E$ have the same support and confidence and should be valid association rules. However, as can be seen from the table, $E$ always appears regardless of whether $G$ appears [20]. Obviously, $G \Rightarrow E$ is not a valid association rule [21].

3.7.2. T-Test. The idea of the $T$-test can be introduced into the measurement of association rules. The test can be introduced in the following ways.

(a) One-Sample T-Test. A one-sample T-test tests if there is a difference between the mean of a single variable and a given constant. For example, the researcher wants to know the difference between the average score of a group of students and the score of 85 . If the population mean is known, the difference significance test between the sample mean and the population mean is also a single sample $T$-test.

Let the overall $X$ obey the normal distribution $N\left(\mu, \sigma^{2}\right)$, where $\sigma^{2}$ is unknown, from which the sample $\left.\left(X_{1}, X_{2}, X_{3}, \ldots, X_{n}\right)\right)$ is extracted; it is now necessary to test the hypothesis $H_{0}:{ }_{\mu}=\mu_{0}, H_{1}:{ }_{\mu} \neq \mu_{0}$, where $\mu_{0}$ is a known constant. When making a hypothesis test on the population mean and when the variance ${ }_{\sigma}^{2}$ is known, choose the statistic:

$$
U=\frac{\left(\bar{X}-\mu_{0}\right)}{(\sigma / \sqrt{n})} \text {. }
$$

But now ${ }_{\sigma}$ is unknown, so you cannot directly use $\mathrm{U}$ as the test statistic. Usually when the standard deviation ${ }_{\sigma}$ is unknown, the subsample standard deviation $S$ is used instead of the overall standard deviation to get a new statistic.

$$
T=\frac{\left(\bar{X}-\mu_{0}\right)}{(S / \sqrt{n})} .
$$

It can be known from the sampling distribution theorem that if $H_{0}$ is established, the statistic $T$ obeys the $t$ distribution with a degree of freedom of $n-1$. For a given $a$, the 
TABLE 1: A set of transaction data.

\begin{tabular}{lc}
\hline TID & Items \\
\hline 1 & $E, G, I, J, M$ \\
2 & $E, F, H, K, L, M, J$ \\
3 & $E, F, G, L, I$ \\
4 & $E, I, J, M$ \\
5 & $E, L, M, K$ \\
6 & $E, F, H, K, L, I, J$ \\
7 & $E, G, I, L, M$ \\
8 & $E, G, L, M$ \\
9 & $E, F, H, I, J, K, L$ \\
10 & $E, F, H, K, L, M, J$ \\
\hline
\end{tabular}

critical value $t_{a}(n-1)$ that can be checked by the $t$ distribution table makes

$$
P\left\{|T|>t_{a}(n-1)\right\}=a .
$$

(b) Independent Sample T-Test. The independent sample Ttest compares the two-sample means, requiring the two samples to be independent of each other, the samples are all from the normal population, and the mean is a descriptive statistic that is meaningful for the test.

The first case: when the two population variances are unknown and equal, $\sigma_{1}=\sigma_{2}$, the combined variance is used as an estimate of the two population variances, mathematically defined as

$$
S_{P^{2}}=\frac{\left(n_{1}-1\right) s_{1}^{2}+\left(n_{2}-1\right) s_{2}^{2}}{n_{1}+n_{2}-2},
$$

where $s_{1}^{2}, s_{2}^{2}$ are the variance of the first and second sets of samples, respectively; $n_{1}, n_{2}$ are the number of samples of the first and second sets of samples, respectively. At this time, the variance $\sigma_{12}^{2}$ of the sampling distribution of the difference between the two samples is

$$
\sigma_{12}^{2}=\frac{S_{P^{2}}}{n_{1}^{2}}+\frac{S_{P^{2}}}{n_{2}^{2}} .
$$

The second case: when the two population variances are unknown and unequal, that is, $\sigma_{1} \neq \sigma_{2}$, respectively, the respective variances are used, and the variance $\sigma_{12}^{2}$ of the sample distribution of the two-sample mean difference is

$$
\sigma_{12}^{2}=\frac{S^{2}}{n_{1}^{2}}+\frac{S^{2}}{n_{2}^{2}}
$$

Thus, the test statistic of the two population mean difference test is $t$ statistic, and the mathematical definition is

$$
t=\frac{\bar{X}_{1}-\bar{X}_{2}-\left(\mu_{1}-\mu_{2}\right)}{\sqrt{\sigma_{12}^{2}}} \text {. }
$$

(c) Paired Sample T-Test. In practice, some data are naturally related in pairs. In order to be necessary, it is sometimes necessary to design experiments so that the data become related pairs. Innocent is naturally related or artificially designed. The variance between the two samples is not equal. At this time, the test of the two population averages with no significant difference is to use the paired sample $T$-test process, and the $T$-test of the paired sample is required to make the two samples compared have a pairing relationship. Both samples are required to come from a normal population, and the mean is a descriptive statistic that is meaningful for the test.

The paired sample $T$-test can be considered as an extension of the one-sample $T$-test, but the test object is the difference between a group of observations from a normal assignment independent sample to a two-group paired sample. If the difference between the two paired samples $x_{1 i}$ and $x_{2 i}$ is $d_{i}=x_{1 i}-x_{2 i}$ independent and comes from the normal allocation, whether the maternal expected value $\mu$ of $d_{i}$ is $\mu_{0}$ can be obtained by the following statistics.

Among them $i=1,2, \ldots \ldots, n, X, Y$, and $Z$ all follow a normal distribution, where $\mu_{z}=E(Z)$ constructs a new statistic:

$$
T=\frac{Z}{S_{i} \sqrt{n-1}} .
$$

If the assumption is true, according to the sampling distribution theorem, the statistic $T$ obeys the $t$ distribution with a degree of freedom of $n=1$. For a given $a$, a critical value can be obtained from the $t$ distribution $t_{a / 2}(n-1)$; then

$$
P\left\{|T|>t_{a / 2}\left(n_{1}+n_{2}-2\right)\right\}=a .
$$

\section{Instance Simulation}

The evaluation of multimedia-assisted piano teaching is not the purpose of evaluation activities. The ultimate goal of evaluation is to improve the work, scientific management, and research decision-making by evaluating the information obtained. The evaluation information data do not spontaneously play a role, and it is necessary to appropriately process the evaluation information, give feedback on the evaluation information, and exert its comprehensive role as much as possible to make the evaluation activity effective. This is also the purpose of multimedia-assisted piano teaching evaluation data analysis. This chapter uses the LIBSVM toolkit and modifies it accordingly and then uses MATLAB to perform multimedia-assisted piano teaching modeling on real datasets.

4.1. Proposed Multimedia-Assistant Piano Teaching (MAPT) Model. The model mainly uses the association rule algorithm in data mining to sort and analyze the data and related data in the teaching evaluation database to obtain the distribution state of the data, the characteristics of the data, the change law of the data, and the model between the data. Provide useful information to improve the quality of teaching and provide decision support to managers. The model function diagram is shown in Figure 3. 


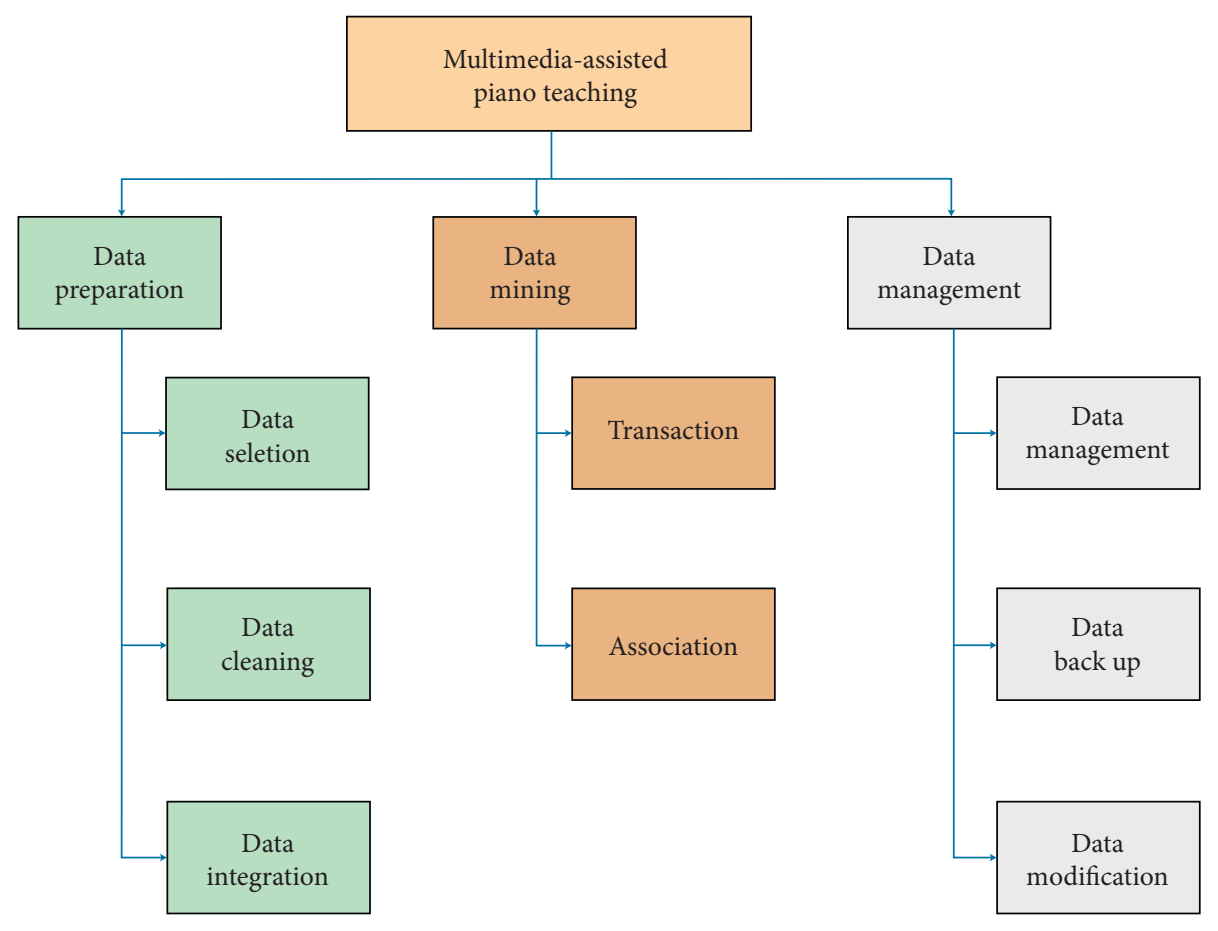

Figure 3: Model function diagram.

4.2. Implementation of Proposed MAPT Model. The model adopts an association rule mining (ARM) algorithm. ARM is a rule-based technique for identifying fascinating relations among variables. The relationship is discovered in the huge dataset. It is envisioned to recognize robust rules. The model adopts Apriori algorithm as the ARM algorithm of the system and adds the function of attribute selection based on Apriori algorithm. Then, in the association rule generation algorithm, the influence measure based on $T$-test is added to the traditional confidence measure, in order to provide more interesting and useful association rules. In the execution process, the algorithm first opens the database, reads the original data in the database, and converts the original data into an instance object by calling the application. Initialize the instance object, and display the attributes in the instance object in the form of a table. The user can select the item of interest and reedit the instance object according to the item selected by the user. Items that are not of interest and records are deleted, and the instance class object is reformed as in Algorithm 1.

4.3. Results Analysis and Discussion. This section provides the detail about results of the proposed model. The data collected at the edges of the network are analyzed using the machine learning approach. The mining algorithm of the above association rules is applied to the evaluation system of MAPT in a university. According to the existing evaluation result data of the school, some association rules can be obtained. Taking all the data of the multimedia-assisted piano teaching effect evaluation of the university from 2005 to 2007 as an example, the association rules of the teacher's title, the teacher's highest age, the teacher's age, and the teacher's multimedia-assisted piano teaching evaluation results are used. Association rule analysis result 1 is shown in Table 2.

(1) Minimum support: 0.3

(2) Number of iterations: 9

(3) The generated large item set:

(4) Size of the big data item $L(1): 11$

(5) The size of the big data item $L(2): 11$

(6) The size of the big data item $L(3): 1$

(7) The best rules have been found.

If we set the minimum confidence level to $80 \%$ and the minimum impact degree to 4 , we can get the association rules "professor, doctoral $\Rightarrow$ excellence" and "professor $\Rightarrow$ excellent." The data of the association rule show that the higher the title, the higher the education and the student evaluation, the better the result, and the quality of the teacher's multimedia-assisted piano teaching has a lot to do with the academic qualifications and professional titles, which shows that the school's teacher training is very important.

The data of the piano teaching effect from 2005 to 2007 are considered as a case study. The evaluation of the multimedia auxiliary piano teaching effect is considered in the case study. The first-level indicators of the multimedia classroom teaching evaluation standard in the multimediaassisted piano teaching effect evaluation system are adapted. The major attributes are teacher quality, teaching design, and teaching process. Then the association rules between the teaching environment and the teacher's multimedia-assisted piano teaching evaluation results are extracted. Before the analysis of association rules, we quantified the evaluation 


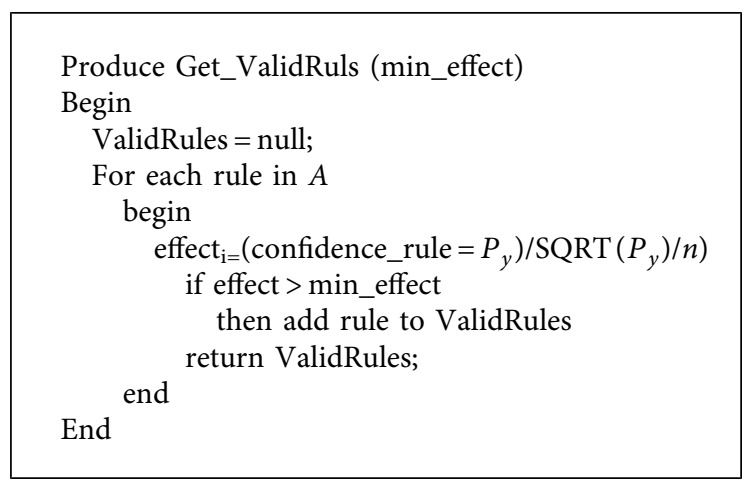

Algorithm 1: Proposed algorithm.

TABLE 2: Association rule analysis result 1.

\begin{tabular}{lcc}
\hline Association rules & Confidence & Influence degree \\
\hline Professor, doctor $\longrightarrow$ excellent & 0.88 & 4.87 \\
Professor $\longrightarrow$ excellent & 0.83 & 4.32 \\
Elderly $\longrightarrow$ professor, excellent & 0.67 & 9.98 \\
Doctor $\longrightarrow$ professor, excellent & 0.52 & 2.16 \\
lecturer $\longrightarrow$ master & 0.69 & 6.87 \\
Doctor, excellent $\longrightarrow$ professor & 0.56 & 3.57 \\
\hline
\end{tabular}

scores of teacher quality, teaching design, teaching process, teaching courseware, and teaching environment. There are four grades of excellent, good, medium, and poor. Association rule analysis result 2 is shown in Table 3 .

(1) Minimum support: 0.3

(2) Number of iterations: 7

(3) The generated large item set:

(4) Size of the big data item $L(1): 12$

(5) The size of the big data item $L(2): 27$

(6) The size of the big data item $L(3): 6$

If we set the minimum confidence level to $70 \%$ and the minimum impact degree to 4 , we can get the association rules "teacher quality (excellent), teaching process (excellent) $\Rightarrow$ excellent" and "teacher quality (excellent) $\Rightarrow$ excellent." The data of the association rule show that the quality of multimedia-assisted piano teaching has a lot to do with the quality of teachers and the teaching process. It should be noted that the "teaching environment" does not appear in the excavated rules. There are two possibilities. The construction of multimedia-assisted piano teaching environment has little effect on the quality of multimediaassisted piano teaching. Secondly, the score of "teaching environment" is too low, which does not promote the quality of multimedia-assisted piano teaching. We believe that the second possibility is very likely to occur, indicating that the construction of multimedia-assisted piano teaching environment is now a weak link, and construction should be stepped up to provide good support for multimedia-assisted piano teaching. Practice has shown that, in the analysis of multimedia-assisted piano teaching evaluation data, the use of association rules technology to dig can obtain useful
TABle 3: Association rule analysis result 2.

\begin{tabular}{lcc}
\hline Association rules & Confidence & Influence degree \\
\hline $\begin{array}{l}\text { Teacher quality (excellent), } \\
\text { teaching process (excellent) } \longrightarrow \\
\text { excellent }\end{array}$ & 0.78 & 9.87 \\
\hline $\begin{array}{l}\text { Teacher quality (excellent) } \longrightarrow \\
\text { excellent }\end{array}$ & 0.63 & 6.32 \\
\hline $\begin{array}{l}\text { Instructional design } \\
\text { (excellent) } \longrightarrow \text { teaching } \\
\text { courseware (excellent) }\end{array}$ & 0.57 & 5.98 \\
\hline $\begin{array}{l}\text { Teacher quality (excellent) } \longrightarrow \\
\text { teaching process (excellent) }\end{array}$ & 0.42 & 3.16 \\
\hline
\end{tabular}

information for teachers' own quality improvement and teaching management decision-making.

The exhaustive and comprehensive experiments are carried out. The dataset of the year 2005 to 2007 of the local university is considered. Experiments were conducted on the validity of the impact of the multimedia teaching evaluation. It is verified whether the use of influence can reduce the generation of redundant rules. The experiment was carried out in Lenovo computer $8 \mathrm{~g}$ memory, $3 \mathrm{G}$ frequency, Win XP, MATLAB environment. The degree of influence is used to filter the association rules generated under the traditional support and confidence framework. When the minimum confidence is set to 0.5 , the total number of association rules generated before and after filtering will be tested with the support threshold. The support degree verification diagram is shown in Figure 4. The scatter plot of support is shown in Figure 5. The scatter diagram of the confidence association rules is shown in Figure 6.

After filtering from the above figure, it can be seen that the number of effective association rules measured by the degree of influence to the association rules generated under the support and confidence framework has been significantly reduced. This shows that the use of influence can effectively negate irrelevant rules and filter out redundant rules. In order to reflect the superiority of the model, the more popular model based on traditional correlation and effectiveness is selected. In order to increase the contrast, the same set of experimental data is selected. Comparison of traditional relevance validity models is shown in Table 4. 


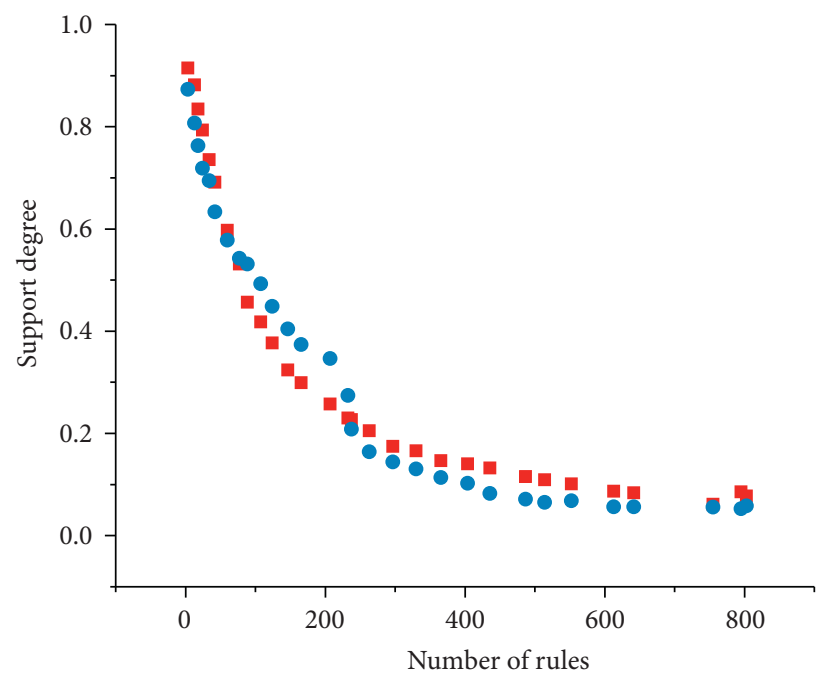

- Category 1

- Category 2

Figure 4: Support degree verification diagram.

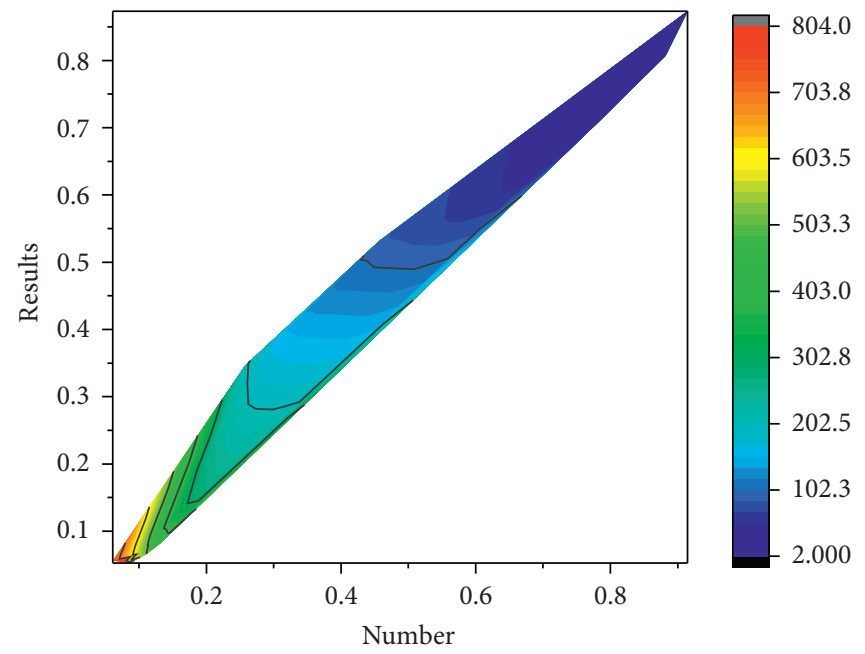

FIGURE 5: Scatter plot of support degree and confidence association rules.

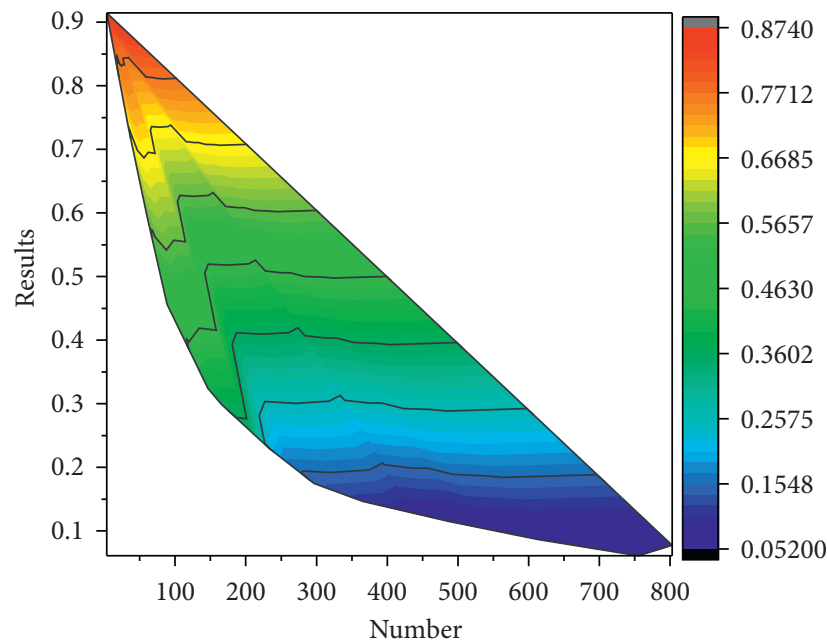

FIgURE 6: The scatter diagram of the confidence association rules. 
TABLE 4: Comparison of traditional relevance validity models.

\begin{tabular}{|c|c|c|c|c|}
\hline Influence degree & Detected influence & Correct number & False detection & Precision rate \\
\hline 8.23 & 8.29 & 8.09 & 0.2 & 0.982989064 \\
\hline 8.28 & 8.34 & 8.14 & 0.2 & 0.983091787 \\
\hline 8.33 & 8.39 & 8.19 & 0.2 & 0.983193277 \\
\hline 8.38 & 8.44 & 8.24 & 0.2 & 0.983293556 \\
\hline 8.43 & 8.49 & 8.29 & 0.2 & 0.983392645 \\
\hline 8.48 & 8.54 & 8.34 & 0.2 & 0.983490566 \\
\hline 8.53 & 8.59 & 8.52 & 0.07 & 0.998827667 \\
\hline 8.58 & 8.88 & 8.81 & 0.07 & 1.026806527 \\
\hline 8.63 & 8.93 & 8.86 & 0.07 & 1.026651217 \\
\hline 8.68 & 8.98 & 8.91 & 0.07 & 1.026497696 \\
\hline 8.98 & 9.28 & 9.21 & 0.07 & 1.025612472 \\
\hline 9.28 & 8.78 & 8.71 & 0.07 & 0.938577586 \\
\hline 9.58 & 9.08 & 9 & 0.08 & 0.939457203 \\
\hline 9.88 & 9.38 & 9.3 & 0.08 & 0.941295547 \\
\hline 9.8 & 9.3 & 9.22 & 0.08 & 0.940816327 \\
\hline 9.72 & 9.22 & 9.14 & 0.08 & 0.940329218 \\
\hline 9.64 & 9.14 & 9.06 & 0.08 & 0.939834025 \\
\hline 9.56 & 9.06 & 8.98 & 0.08 & 0.939330544 \\
\hline 9.48 & 8.98 & 8.9 & 0.08 & 0.938818565 \\
\hline
\end{tabular}

Table 4 describes the detail of the traditional relevance in the context of validity. The validation is achieved and depicted in Table 4.

The experimental results show that the use of influence can not only obtain effective association rules but also divide the obtained rules into strong association rules and weak association rules and overcome some deficiencies of relevance and validity, making the rules more objective, reasonable, and convenient for users to choose. The verification results show that it is feasible and valuable to find the potential relevance.

\section{Conclusion}

This paper studies and analyzes the traditional support and confidence framework based on edge-enabled data analysis. The metrics such as relevance and validity indicate the shortcomings. On this basis, the T-test is introduced into the measurement of association rules of machine learning, and a new measure of the relevance of association rules is proposed. The model is utilized to assess the potential correlation of piano teaching using machine learning techniques. The ARM technique of machine learning is utilized with the integration of improved $T$-test method. The data analysis is performed at the edges of network for efficient results. While the theoretical and visual analysis of the impact metric is carried out, its effectiveness is verified by an example. The machine-related association rule metric impact is applied to the analysis of multimedia-assisted piano teaching quality evaluation data. Effectively improve the reliability, scalability, reusability, and maintainability of the model. The feasibility and effectiveness of the proposed framework are verified using the analysis of the actual dataset. The verification results show that it is feasible and valuable to find the potential relevance of multimedia-assisted piano teaching evaluation data by using the degree of influence as the measure of association rules. It is evident from the results that the use of the degree of influence as a measure of association rules to find the potential relevance of multimediaassistant piano teaching evaluation data is extremely feasible.

\section{Data Availability}

The data used to support the findings of this study are available from the corresponding author upon request.

\section{Conflicts of Interest}

The authors declare that they have no conflicts of interest.

\section{Acknowledgments}

This study was supported by 2019 Key Projects of Art and Science Planning in Heilongiiang (2019A003).

\section{References}

[1] H. Sarac, "Effect of multimedia assisted 7e learning model applications on academic achievement and retention in students," European Journal of Educational Research, vol. 6, no. 3, pp. 299-311, 2017.

[2] L. Tan, C. Chen, Z. F. Fang et al., "Exploration of multimediaassisted and bilingual teaching model in analytical chemistry experiments," Laboratory Science, vol. 8, no. 5, pp. 221-235, 2017.

[3] O. Ji-Won, O. S. Nam, I. Choi Joon et al., "Does the gadoxetic acid-enhanced liver MRI impact on the treatment of patients with colorectal cancer? comparison study with18F-FDG PET/ CT," BioMed Research International, vol. 1, pp. 841-857, 2016.

[4] R. S. Florêncio, M. S. B. Meira, M. V. D. Cunha et al., "Plasmatic estradiol concentration in the mid-luteal phase is a good prognostic factor for clinical and ongoing pregnancies, during stimulated cycles of in vitro fertilization," Jbra Assisted Reproduction, vol. 22, no. 1, pp. 8-14, 2018.

[5] A. Moglia, V. Perrone, V. Ferrari et al., "Influence of videogames and musical instruments on performances at a 
simulator for robotic surgery," Minimally Invasive Therapy \& Allied Technologies, vol. 26, no. 3, pp. 129-134, 2017.

[6] M. Bartoli, L. Rosi, A. Giovannelli et al., "Production of biooils and bio-char from Arundo donax, through microwave assisted pyrolysis in a multimode batch reactor," Journal of Analytical \& Applied Pyrolysis, vol. 122, 2016.

[7] J. Lv, "Research of Japanese translation teaching based on multimedia network technology," Journal of Computational and Theoretical Nanoscience, vol. 13, no. 12, pp. 10375-10379, 2016.

[8] P. Zhou, Y. Zhou, D. Wu, and H. Jin, "Differentially private online learning for cloud-based video recommendation with multimedia big data in social networks," IEEE Transactions on Multimedia, vol. 18, no. 6, pp. 1217-1229, 2016.

[9] L. I. Mei and X. N. University, "Teaching reform research based on assisted multimedia of tourism management major," Education Teaching Forum, vol. 19, no. 2, pp. 122-135, 2017.

[10] E. Akin, "Observation of multimedia-assisted instruction in the listening skills of students with mild mental deficiency," Educational Research \& Reviews, vol. 11, no. 5, pp. 182-193, 2016.

[11] N. Zaranis, "The use of ICT in kindergarten for teaching addition based on realistic mathematics education," Education and Information Technologies, vol. 21, no. 3, pp. 589-606, 2016.

[12] J. Li, R. Singh, and R. Singh, "A novel large-scale multimedia image data classification algorithm based on mapping assisted deep neural network," Multimedia Tools \& Applications, vol. 76, no. 18, pp. 1-24, 2017.

[13] H. Tambunan and E. Napitupulu, "Effectiveness of interactive multimedia based learning model in engineering mechanics," International Education Studies, vol. 9, no. 10, p. 155, 2016.

[14] Y. Bai, Z. Chen, J. Xie, and C. Li, "Daily reservoir inflow forecasting using multiscale deep feature learning with hybrid models," Journal of Hydrology, vol. 532, pp. 193-206, 2016.

[15] J. Yang, B. Champagne, Y. Zou, and L. Hanzo, "Joint optimization of transceiver matrices for MIMO-aided multiuser AF relay networks: improving the QoS in the presence of CSI errors," IEEE Transactions on Vehicular Technology, vol. 65, no. 3, pp. 1434-1451, 2016.

[16] F. Wang, J. Liu, M. Chen, and H. Wang, "Migration towards cloud-assisted live media streaming," IEEE/ACM Transactions on Networking, vol. 24, no. 1, pp. 272-282, 2016.

[17] K.-H. Chiang, C.-Y. Fan, H.-H. Liu, and G.-D. Chen, "Effects of a computer-assisted argument map learning strategy on sixth-grade students' argumentative essay reading comprehension," Multimedia Tools and Applications, vol. 75, no. 16, pp. 9973-9990, 2016.

[18] D. Fan, L. Wei, and M. Cao, "Extraction of target region in lung immunohistochemical image based on artificial neural network," Multimedia Tools \& Applications, vol. 75, no. 19, pp. 1-18, 2016.

[19] F. Korur, S. Toker, and A. Eryılmaz, "Effects of the integrated online advance organizer teaching materials on students' science achievement and attitude," Journal of Science Education and Technology, vol. 25, no. 4, pp. 628-640, 2016.

[20] S. Kim, "Behavioral learning game for socio-physical IoT connections," Eurasip Journal on Wireless Communications \& Networking, vol. 2016, no. 1, pp. 1-11, 2016.

[21] R. Boateng, S. L. Boateng, R. B. Awuah et al., "Videos in learning in higher education: assessing perceptions and attitudes of students at the University of Ghana," Smart Learning Environments, vol. 3, no. 1, pp. 1-13, 2016. 